\title{
Contents, Vol. 193, 1986
}

Vol. 193, 1986

Index Vol. 193,1986

No. 1-2 Original Paper $\cdot$ Travaux originaux $\cdot$ Originalarbeiten

Topographisch-chirurgische Anatomie der kindlichen Pars plana

Dietl, R.H.; Stefani, F.H 1

Preliminary Research on the Resistance of IOLs to a Q-Switched Nd-YAG Laser (With 1 color plate)

Balacco-Gabrieli, C; Palmisano, C; Castellano, L 10

Hemispheric Retinal Branch Vein Occlusions

Gómez-Ulla de Irazazabal, F.J.; Cadarso Suarez, L.; Orduña Domingo, E 14

Inflamed Eye in Marfan's Syndrome with Posteriorly Luxated Lens

Croxatto, J.O.; Lombardi, A.; Malbran, E.S 23

Topical Retinoic Acid Treatment of Drug-Induced Pseudopemphigoid (With 1 color plate)

Herbort, C.P.; Matter, M.; Uffer, S 27

Management of Purulent Postoperative Endophthalmitis

Laatikainen, L.; Tarkkanen, A

34

Sclerosing Orbital Pseudotumor

Cervellini, P.; Volpin, L.; Curri, D.; Pedrotti, M.; Benedetti, A

Mechanical Behavior of the Sclera

Arciniegas, A.; Amaya, L.E 45

The Normal Visual Field on the Humphrey Field Analyzer

Brenton, R.S.; Phelps, CD 56

Significance of Postgamma Protein Fraction in Tears

Suárez, J.C 75

Klinische Intepretation retinaler Durchblutungsmessungen. I. Messtechnische Möglichkeiten und klinische Intepretationshilfe

Vilser, W.; Königsdörffer, E.; Jütte, A.; Deufrains, A

Klinische Intepretation retinaler Durchblutungsmessungen. II. Blutfluss

Vilser, W.; Seewald, D.; Dietze, D.; Friedrich, R.; Deufrains, A.; Königsdörffer, E.;

Jütte, A 88

Klinische Intepretation retinaler Durchblutungsmessungen. III. Blutgeschwindigkeit und

Gefássdurchmesser bei Normalpersonen und bei Patienten mit venösen Verschluss-

krankheiten

Vilser, W.; Gräser, T.; Leisner, H.; Deufrains, A.; Königsdörffer, E.; Seewald, D.;

Friedrich, R.; Jütte, A 97

Klinische Intepretation retinaler Durchblutungsmessungen. IV. Fotokoagulation bei diabetischer Retinopathie und venösen Verschlusserkrankungen

Vilser, W.; Deufrains, A.; Dietze, U.; Seewald, D.; Büchner, D.; Königsdörffer, E.; Jütte, A 108 
Book Reviews $\cdot$ Livres nouveaux $\cdot$ Buchbesprechungen 126

No. 3 Original Paper · Travaux originaux — Originalarbeiten

Sphäroidale Degeneration der Hornhaut

Sayar, R.B.; Domains, D. von; Steinhorst, U 129

IV Index

Equipement optique des sujets atteints de dégénérescence maculaire senile

exsudative.Comparaison des cicatrices fibrogliales spontanées et des scotomes induits par photocoagulationBeuchat, L.; Pournaras, C.J 135

Congenital Retinal Macrovessel

Crecchio, G. de; Mastursi, B.; Alfieri, M.C., Pignalosa, B 143

Effect of Timolol on Optic Nerve Head Autoregulation

Pillunat, L.E.; Stodtmeister, R.; Wilmanns, I.; Metzner, D . 146

Immunohistochemical Study of Embryonic Rhabdomyosarcomas

Garrido, CM.; Arra, A154

Clinicopathologic Case Report · Description clinico-patholologique de cas · Klinisch-

pathologischeFallbeschreibung

Malignes Lymphom der Lider

Witschel, $\mathrm{H} \quad 161$

Abstract

$\mathrm{S}$

Netherlands Ophthalmological Society

180th Annual Meeting, March 18-20, 1986, Apeldoorn

169

Book Review · Livre nouveau · Buchbesprechung 192

Announcement $\cdot$ Communication · Ankündigung 192

No. 4 Original Paper $\square$ Travaux originaux $\cdot$ Originalarbeiten

Superficial Involvement in Lattice Corneal Dystrophy

Zechner, E.M.; Croxatto, J.O.; Malbran, E.S.193

Localized Chorioretinal Degeneration in Patients Receiving Hemodialysis

Kitaoka, M.; Hayssaka, S.; Mizuno, K 200

Hereditary Congenital External Ophthalmoplegia

Houtman, W.A.; Weerden, T.W. van; Robinson, P.H.; Vries, B. de; Hoogenraad, Tj.U. 207 La

sarcoïdose des voies optiques. Discussion diagnostique

Sahel, J.; Flament, J.; Buchheit, F.; Bronner, A 219

Orbitocranial Fibrous Dysplasia. A Case Report

Yamaguchi, K.; Hayasaka, S.; Yamada, T.; Yokoyama, T.; Kameyama, M 225

Contrast Echography of the Eye and Orbit

Miszalok, V.; Fritzsch, T.; Wollensak, J .231

Untersuchungen zur medikamentösen Beeinflussung der Sulfhydryloxidation in isolierten

Kaninchenlinsen

Elstner, E.F.; Heinisch, H.; Kroner, R.; Adamczyk, R 236

Studies of Ocular Retinoblastomas with Immunoperoxidase Techniques

Garrido, CM.; Arra, A. 242

Scintimetric Detection of Choroidal Malignant Melanoma with [123I]-5-Iodo-2-Thiouracil

Franken, N.A.P.; Delft, J.L. van; Langevelde, A. van; Bleeker, J.C; Wolff-Rouendaal,

D. de; Best, J.A. van; Bouwhuis-Hoogerwerf, M.L.; Oosterhuis, J.A.; Pauwels, E.K.J. . 248

Book Reviews · Livres nouveaux — Buchbesprechungen 255 
A. Author Index

258

Subject Index 259 